\title{
Differences in Genetic Background Contribute to Pseudomonas Exotoxin A-Induced Hepatotoxicity in Rats
}

\author{
Chien-Chao Chiu ${ }^{1,+}{ }^{+}$Yu-Chih Wang ${ }^{2,+}$, Wen-Ching Huang ${ }^{3}$, Yi-Hsun Chen ${ }^{2}$, Shao-Wen Hung ${ }^{1}$, \\ Yen-Te Huang ${ }^{4}$, Hsiao-Li Chuang ${ }^{4}$ (D) and Yi-Chih Chang ${ }^{5, *}$
}

1 Animal Technology Laboratories, Agricultural Technology Research Institute, Miaoli 350, Taiwan; chiu2295@yahoo.com.tw (C.-C.C.); $1032169 @$ mail.atri.org.tw (S.-W.H.)

2 Graduate Institute of Veterinary Pathobiology, College of Veterinary Medicine,

National Chung Hsing University, Taichung 402, Taiwan; joelovefu@gmail.com (Y.-C.W.);

u9423201@gmail.com (Y.-H.C.)

3 Department of Exercise and Health Science, National Taipei University of Nursing and Health Sciences, Taipei 112, Taiwan; magicpica521@gmail.com

4 National Laboratory Animal Center, National Applied Research Laboratories, Taipei 115, Taiwan; evan@nlac.narl.org.tw (Y.-T.H.); p650214@nlac.narl.org.tw (H.-L.C.)

5 Department of Medical Laboratory Science and Biotechnology, China Medical University,

Taichung 404, Taiwan

* Correspondence: yichih@mail.cmu.edu.tw; Tel.: +886-4-2205-3366\#7209

+ These authors contributed same to this work.

Academic Editor: Vernon L. Tesh

Received: 17 May 2017; Accepted: 13 July 2017; Published: 15 July 2017

\begin{abstract}
Pseudomonas aeruginosa exotoxin A (PEA) causes severe hepatotoxicity in experimental animals and is useful in investigations of immune-mediated liver injury. However, strain differences in the sensitivity to PEA-induced hepatotoxicity in rats remains be elucidated. In this study, we determined the severity of PEA-induced hepatotoxicity in six genetically different rat strains. Male LE (Long Evans), Wistar, F344, WKY, BN/SsN and LEW rats were administered a single intravenous injection of PEA $(20 \mu \mathrm{g} / \mathrm{kg})$. Significantly elevated serum ALT and AST levels, massive necrosis and hemorrhage, and numerous TUNEL-positive hepatocytes were observed in BN/SsN rats. In contrast, low levels of ALT and AST as well as mild changes in liver histopathology were observed in Wistar and F344 rats. Moderate levels of hepatic injuries were observed in LE, WKY, and LEW rats. Pro-inflammatory cytokines including TNF- $\alpha$, IL-2 and IL-6 serum levels were markedly increased in $\mathrm{BN} / \mathrm{SsN}$ rats compared to Wistar and F344 rats. However, the hepatic levels of low density lipoprotein receptor-related protein (LRP), which functions as the PEA receptor, were not significantly different in each strain. Taken together, we suggest that $\mathrm{BN} / \mathrm{SsN}$ is the most sensitive rat strain, whereas Wistar and F344 were the most resistant rat strains to PEA-induced liver damage. The different genetic background of rat strains plays an important role in the susceptibility to PEA-induced epatotoxicity that may depend on immune-regulation but not LRP receptor levels.
\end{abstract}

Keywords: Pseudomonas exotoxin A; genetic background; hepatotoxicity; massive necrosis

\section{Introduction}

Pseudomonas aeruginosa is an opportunistic, non-fermentative, Gram-negative rod bacterium. $P$. aeruginosa has emerged as a major infectious disease agent, particularly in patients with burn injuries or cystic fibrosis [1,2]. Several virulence factors, such as P. aeruginosa exotoxin A (PEA) and exoenzyme S, are involved in the disease caused by this bacterium [3]. Previous studies indicated that 
the low-density lipoprotein receptor-related protein (LRP) functions as the receptor that PEA utilizes to gain access to mammalian cells [4]. In addition, the decreased expression of LRP may enhance macrophage and hepatocyte cell-line resistance to PEA induced cytotoxicity [5,6]. Laithwaite and collaborators reported that increased PEA sensitivity in BNL 1ME A7R.1 transformed hepatocytes was associated with increased functional cell surface LRP expression [7].

To date, PEA has been used to establish an experimental animal model for immune-mediated liver injury [8]. In this model, PEA induces an excessive activation of host immune cells (especially Kupffer cells and T cells) that secrete pro-inflammatory cytokines, such as tumor necrosis factor $\alpha$ (TNF- $\alpha$ ), interleukin (IL)-2 and IL-6, resulting in hepatocyte damage [8]. In our previous studies, we treated Wistar and Long-Evans (LE) rats with $20 \mu \mathrm{g} / \mathrm{kg}$ PEA and showed that Wistar rats were more resistant to PEA-induced liver injury than LE rats [8,9]. In other liver injury models, C57BL/ 6 mice have been reported to be resistant to Concanavalin A (ConA)-induced hepatitis, while BALB/c mice were susceptible. The difference in susceptibility between these mouse strains was associated with the development of a hepatitis that depends on IFN- $\gamma$ production levels (high in C57BL/ 6 and low in BALB/c) [10]. In acetaminophen-induced hepatotoxicity models, significant hepatic hemorrhage was observed in $\mathrm{C} 3 \mathrm{He} / \mathrm{FeJ}$ and $\mathrm{CD}-1$ mouse strains, but not in the C57BL/6 mouse strain [11]. These observations could be explained by differences in cytochrome P450 2E1 (CYP2E1) expression in sinusoidal endothelial cells, which has been shown to correlate with the susceptibility to vascular injury and hemorrhage [12]. On the other hand, C57BL/6 mice exhibited high deviations in the development of steatosis and inflammation in response to diet-induced features of non-alcoholic/alcoholic fatty liver disease [13]. Surprisingly, CD-1 mice did not show significant features of fatty liver disease in response to dietary regimens.

Previous studies have observed the development of severe, chronic asthma in a susceptible Brown Norway (BN) rat strain (BN/SsN), but not in a non-susceptible Fischer 344 (F344) strain [14,15]. Hines et al. indicated that pronounced macrophage and mast cell responses developed and persisted in BN/SsN, but not in F344 rats [15]. Although the genetic background is expected to affect the PEA-induced liver damage in different rat strains, this issue has not been sufficiently addressed in the literature. Thus, the current study was conducted to determine the differences in sensitivity to PEA-induced hepatotoxicity between four different inbred rat strains and two outbred stocks, namely F344, Wistar Kyoto (WKY), BN/SsN, Lewis (LEW), LE and Wistar by evaluating their clinical chemistry, liver histopathology and TUNEL staining. Moreover, in the liver LRP protein levels and quantification of pro- and anti-inflammatory cytokines in the serum were performed to determine if the levels of LRP receptors or inflammatory cytokines correlate with the differences in sensitivity to PEA-induced liver injury observed between rat strains.

\section{Results}

\subsection{Hepatic Concentration of LRP in Different Rat Strains}

In order to elucidate the underlying mechanisms for PEA-hepatotoxicty in different rat strains, we reasoned that disease susceptibility may be related to differences in LRP expression or immune-regulation. We firstly evaluated the hepatic expression of LRP in different rat strains. However, no significant difference in LRP expression levels were observed in liver extract from each rat strain without PEA treatment (Figure 1). 


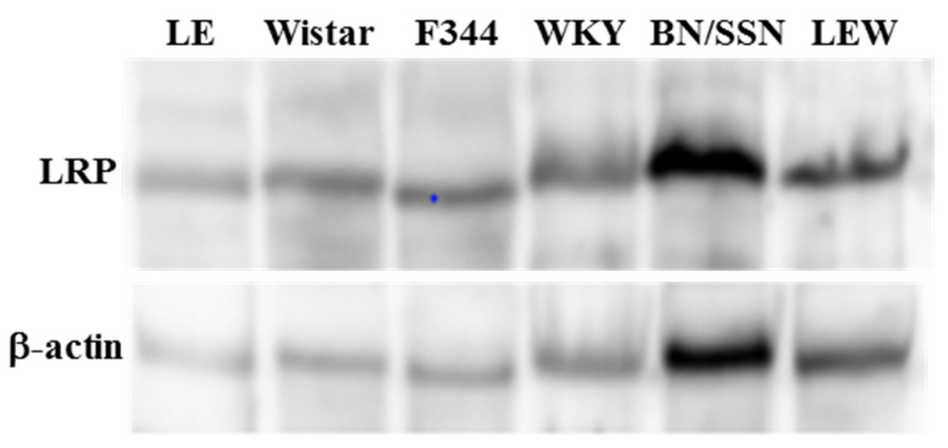

Figure 1. Protein levels of hepatic LRP in different rat strains. The membrane was stripped and immunoblotted with anti- $\beta$-actin antibody a protein loading control.

\subsection{Clinical Chemistry and Complete Blood Count (CBC) Analysis}

According to our previous report, the administration of $20 \mu \mathrm{g} / \mathrm{kg}$ PEA did not induce death of young adult Wistar and LE rats within the first $48 \mathrm{~h}$ post administration [8,9]. Thus, $48 \mathrm{~h}$ after PEA treatment, the serum ALT levels in LE, Wistar, F344, WKY, BN/SsN and LEW rats were analyzed and determined to be $5149 \pm 2230.1 \mathrm{U} / \mathrm{L}, 2649 \pm 937.2 \mathrm{U} / \mathrm{L}, 3312 \pm 1022.6 \mathrm{U} / \mathrm{L}, 5124 \pm 1255.1 \mathrm{U} / \mathrm{L}$, $11032 \pm 4587.2 \mathrm{U} / \mathrm{L}$ and $6992 \pm 1788.4 \mathrm{U} / \mathrm{L}$, respectively (Figure 2). Moreover, the fold changes of ALT compared with basal levels of each rat strains were $88 \pm 34.1,49.2 \pm 16.1,50.7 \pm 14.3,87.7 \pm 16.6$, $180 \pm 68.3$ and $127.4 \pm 29.1$, respectively (Figure S1).
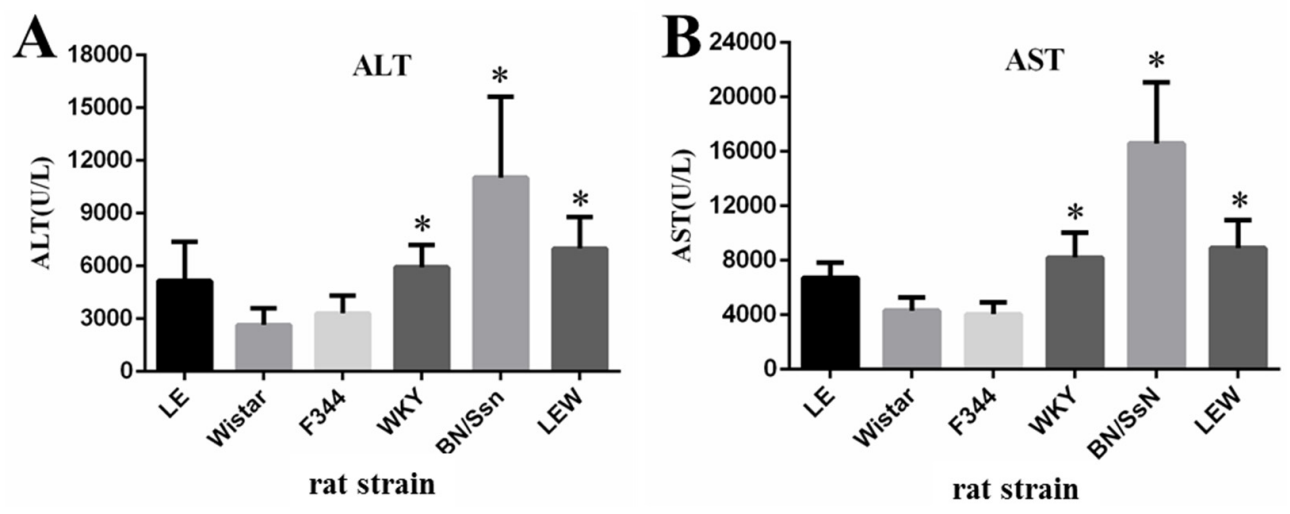

Figure 2. Serum levels of (A) ALT and (B) AST in the PEA-treated different rat strains. All rat strains including LE, Wistar, F344, WKY, BN/SsN and LEW were given PEA intravenously a dose of $20 \mu \mathrm{g} / \mathrm{kg}$ at $48 \mathrm{~h}$. Results are expressed as mean $\pm \mathrm{SD} .{ }^{*} p<0.05$ compared with Wistar rats. Each group animal number $=8$.

A similar trend, as well as fold changes in the serum levels for AST, were also observed between the different rat strains. Notably, ALT and AST serum concentrations were significantly greater in $\mathrm{BN} / \mathrm{SsN}$ rats than in other species, while the lowest serum levels were observed in Wistar and F344 rat strains.

As shown in Figure 3, the total white blood cell (WBC) count was markedly increased in the $\mathrm{BN} / \mathrm{SsN}$ group compared to that in other groups. Meanwhile, total WBC counts were lower in the LE, WKY, and LEW rats compared to those in BN/SsN rats, but still higher than those for Wistar and F344 rats. In comparison, no significant differences between groups were observed for the total red blood cell (RBC) counts. The treatment of all rat strains with Dulbecco's phosphate-buffered saline (DPBS) did not cause ALT and AST elevations, and showed no significant changes in CBC (Figure S1, Table S1). 

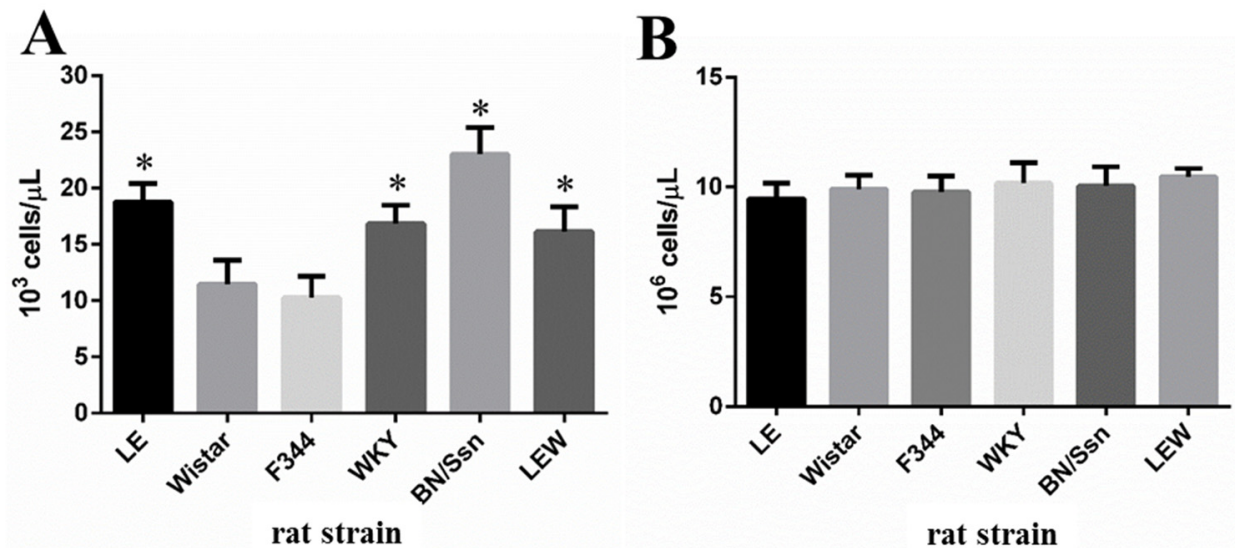

Figure 3. Effect of $20 \mu \mathrm{g} / \mathrm{kg}$ PEA on hematological characteristics of different rat strains (A) WBC; (B) RBC. Results are expressed as mean $\pm \mathrm{SD}$. ${ }^{*} p<0.05$ compared with Wistar rats.

\subsection{Histopathology and TUNEL Staining}

Analysis of histologic liver sections from PEA-treated $\mathrm{BN} / \mathrm{SsN}$ rats showed diffuse, massive necrosis, hepatocyte nuclear condensation, hemorrhage, and dissociation of hepatic cords. In sections of LE, WKY and LEW rats, numerous apoptotic bodies and moderate hepatocyte necrosis were observed. Interestingly, only a small number of apoptotic bodies and mild hepatocyte necrosis were found in histological sections of Wistar and F344 rats (Figure 4). The mean liver injury scores (on a scale from 0 to 5) were calculated for the different rat strains and determined to be for LE: $2.63 \pm 0.92$, Wistar: $1.51 \pm 0.76$, F344: $1.25 \pm 0.46$, WKY: $2.25 \pm 0.88, \mathrm{BN} / \mathrm{SsN}: 4.37 \pm 0.74$ and LEW: $3.12 \pm 0.83$.
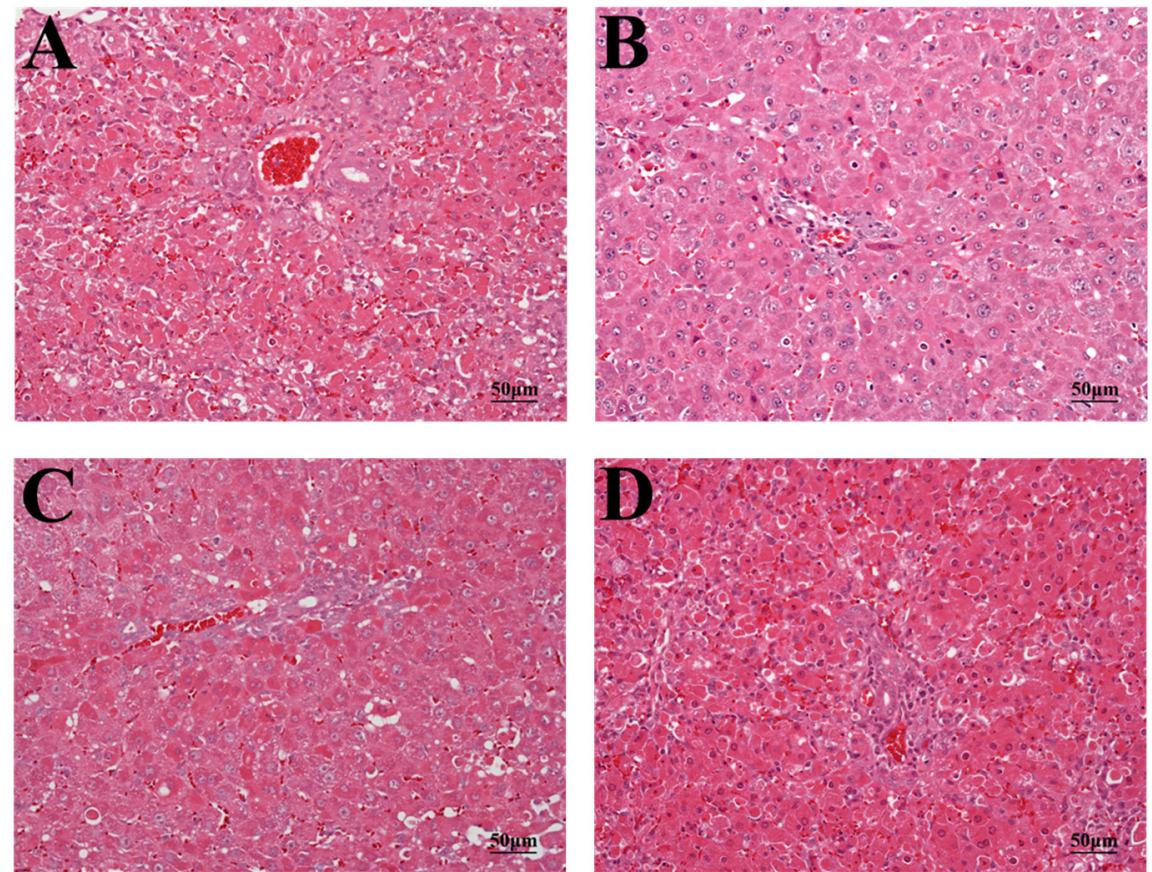

Figure 4. Cont. 

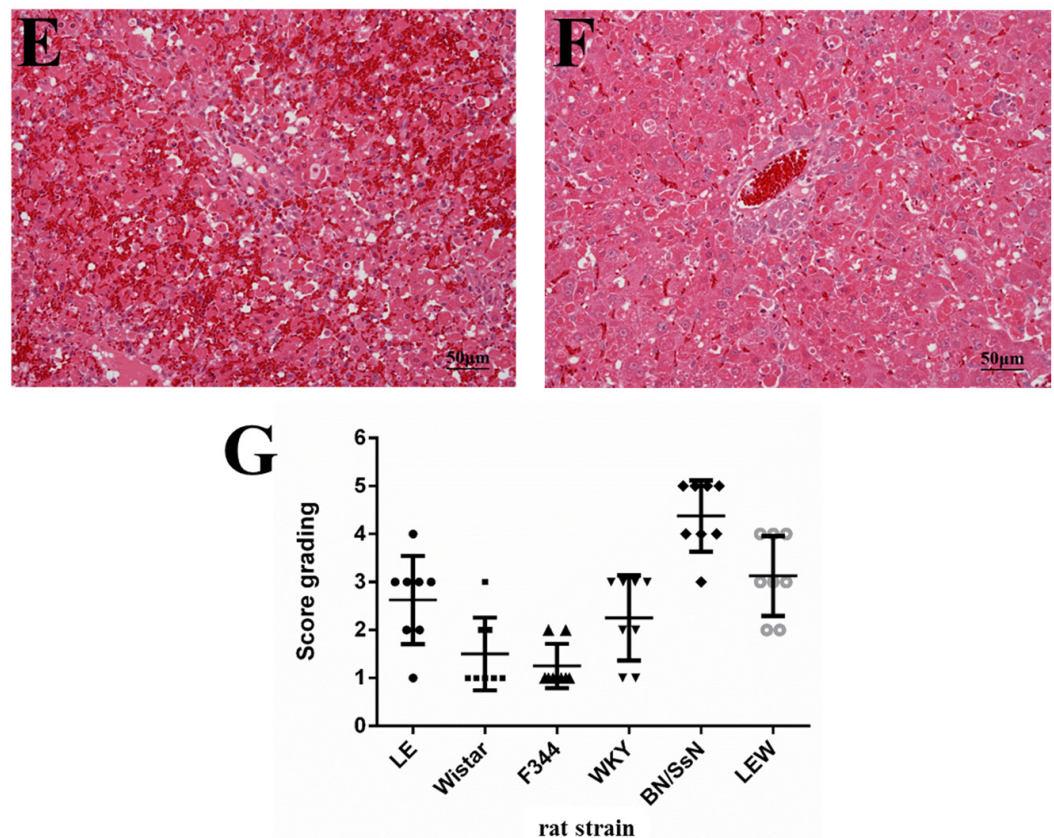

Figure 4. The photomicrographs of liver tissues from rats treated with $20 \mu \mathrm{g} / \mathrm{kg}$ PEA at $48 \mathrm{~h}$. (A) LE; (B) Wistar; (C) F344; (D) WKY; (E) BN/SSN; (F) LEW; (G) Histologic score was combined hepatic lesions and hemorrhage grading (mean $\pm \mathrm{SD}$ ). H\&E stain, magnification $\times 200$.

Treatment with $20 \mu \mathrm{g} / \mathrm{kg}$ PEA caused the presence of a significant number of apoptotic nuclei in the livers of $\mathrm{BN} / \mathrm{SsN}$ rats. In contrast to $\mathrm{BN} / \mathrm{SsN}$ rats, the numbers of TUNEL-positive cells were markedly lower in liver sections of LE, WKY, and LEW rats. Only a few TUNEL-positive cells were observed in liver sections of Wistar and F344 rats (Figure 5). The average number of TUNEL-positive hepatocytes (per 100 $\times$ field) present were calculated to be for LE: $1.62 \pm 0.71$, Wistar: $0.87 \pm 0.64$, F344: $0.74 \pm 0.69$, WKY: $1.53 \pm 0.75$, BN/SsN: $2.67 \pm 0.74$ and LEW: $1.75 \pm 0.88$. No histologic changes or TUNEL-positive cells were observed in the liver samples from DPBS-treated different rat strains (Table S1).
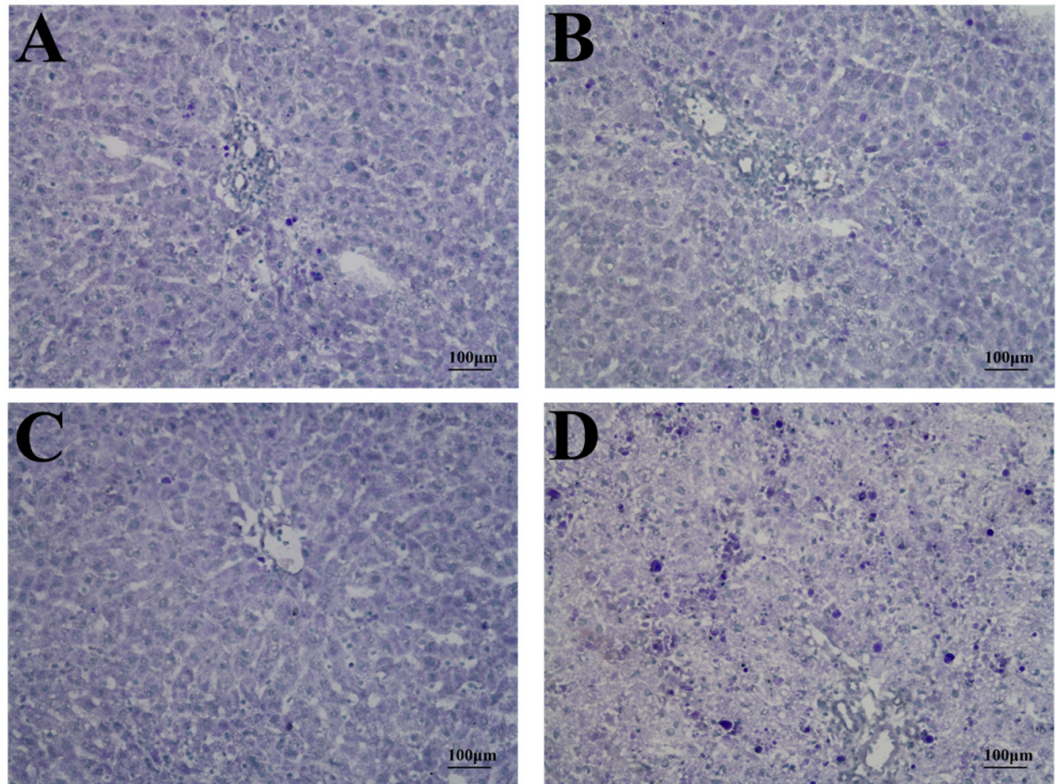

Figure 5. Cont. 

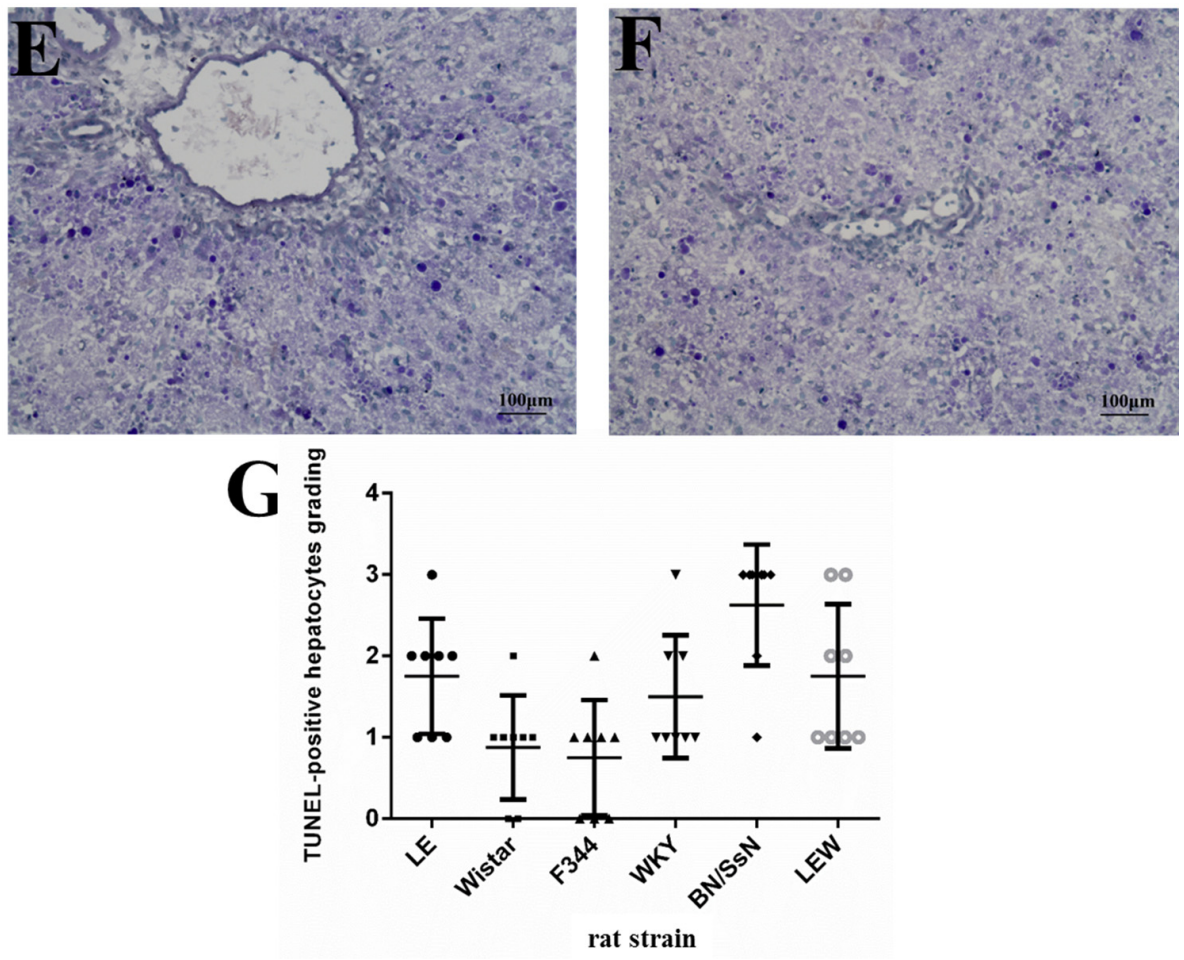

Figure 5. Evaluation of apoptotic hepatocytes by TUNEL assay in the PEA-treated different strain rats at 48 h. (A) LE; (B) Wistar; (C) F344; (D) WKY; (E) BN/SSN; (F) LEW; (G) TUNEL score, which was dependent on the number of TUNEL-positive cells counted per $100 \times$ field (mean \pm SD). TUNEL stain, magnification $\times 200$.

\subsection{Serum Cytokine Levels}

Serum cytokine levels are summarized in Figure 6 . BN/SsN rats had significantly higher TNF- $\alpha$ serum levels compared to those in other rat strains. Moderate TNF- $\alpha$ serum levels were observed in LE, WKY and LEW rats. Only trace amounts of TNF- $\alpha$ were detected in F344 rats. The highest levels of serum IL-2 were found in BN/SsN rats. Serum IL-2 concentrations in Wistar, F344, and WKY rats were significantly lower than those in BN/SsN rats. In LE and LEW rats, the levels of serum IL-2 were moderate. Levels IL-6 in serum were significantly higher in $\mathrm{BN} / \mathrm{SsN}$ rats compared to those in the LE, WKY and LEW rats. Interestingly, only low levels of IL-6 were detected in Wistar and F344 rats. $\mathrm{BN} / \mathrm{SsN}$ rats exhibited significantly higher IL-10 levels in serum when compared to other groups. Meanwhile, the lower levels of IL-10 were noted in F344 rats. Serum TNF- $\alpha$, IL-2, IL-6, and IL-10 were not detected in any of the DPBS-treated rat strains (Table S1).

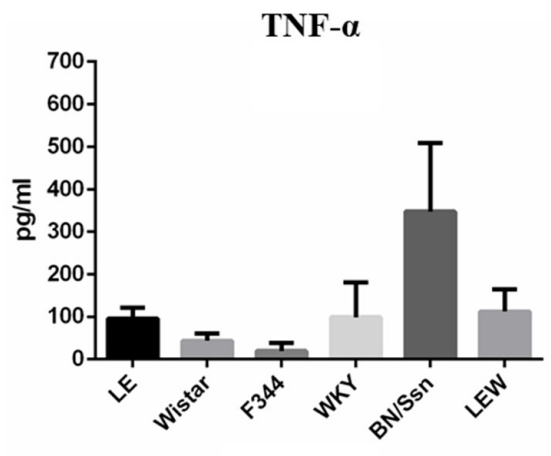

rat strain

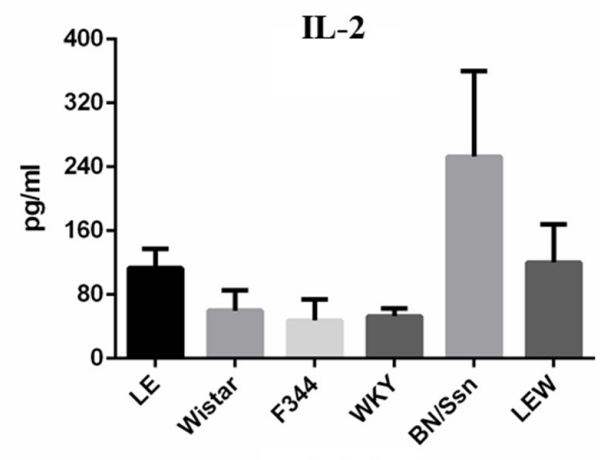

rat strain

Figure 6. Cont. 

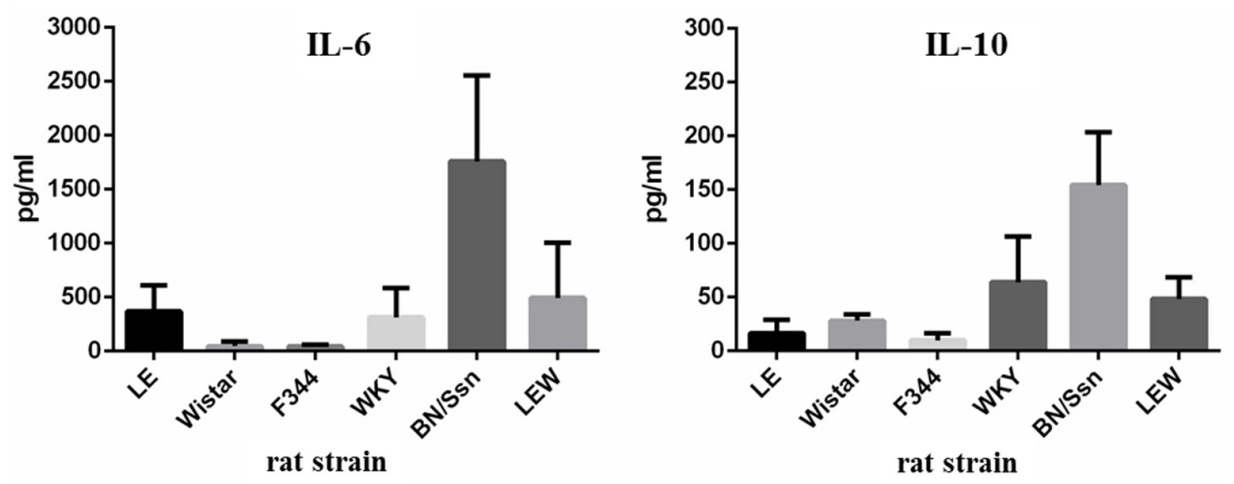

Figure 6. Serum cytokine profiles, including TNF- $\alpha$, IL-2, IL-6 and IL-10 levels from rat strains challenged with $20 \mu \mathrm{g} / \mathrm{kg}$ PEA for $48 \mathrm{~h}$. Results are expressed as mean $\pm \mathrm{SD}$.

\section{Discussion}

The objective of this investigation was to better understand different stocks (LE and Wistar) and strains (F344, WKY, BN/SsN and LEW) of rats in their susceptibility or resistance to a sub-lethal dose of PEA-induced liver injury. The present study showed that BN/SsN rats had severe liver injury in response to treatment with a sub-lethal dose of PEA, while moderate liver damage developed in LE, WKY, and LEW rats. In contrast, PEA treatment induced only low numbers of apoptotic and necrotic hepatocytes in both Wistar and F344 rats. These differences in hepatotoxicity are observed not only with a sub-lethal dose of PEA as shown herein, but also with other mechanistically different models of T-cell-dependent liver injury after treatment with Concanavalin A, acetaminophen or lipopolysaccharide (LPS) treatment $[10,11,16,17]$. Importantly, these results can serve as reference data relating to PEA treatment in different rat genetic backgrounds, which may prove useful for PEA-based anti-tumor drug development.

The present results revealed significant differences in the response of several rat strains to PEA administration. Clinical chemistry values in the blood for ALT and AST were significantly higher in $\mathrm{BN} / \mathrm{SsN}$ rats compared with other strains. The lowest levels of ALT and AST were noted in Wistar and F344 rats. On the other hand, morphological features in of liver damage that included hepatic cord dissociation, liver hemorrhage, and massive hepatocytes necrosis were apparent in BN/SsN rats. A moderate level of hepatocyte necrosis and apoptosis were observed in LE, WKY and LEW rats, whereas only a small number of apoptotic bodies and single cell necrosis were observed in Wistar and F344 rats. These findings are similar to those of previous reports, which show differences in PEA-induced hepatotoxicity in different mouse strains [18-20]. Our data suggests that BN/SsN rats were more sensitive to PEA treatment than Wistar and F344 strains. Although the only one dose $(20 \mu \mathrm{g} / \mathrm{kg})$ and time point $(48 \mathrm{~h})$ was performed in these different rat strains. However, our previous results found that the severity of PEA-induced liver injury was dose and time dependent in Wistar and LE rats $[8,9]$.

We have previously demonstrated that Kupffer cells are capable of producing large amounts of TNF- $\alpha$, and thus can cause severe liver injury, suggesting an important role for hepatic Kupffer cells in PEA-induced hepatotoxicity [8]. It has been shown that gadolinium chloride ( $\mathrm{GdCl} 3$ ) can ameliorate pro-inflammatory cytokine production, as demonstrated in our previous study [21]. In this study, among all the rat strains tested, the highest levels of TNF- $\alpha$ were detected in BN/SsN rats. This finding is consistent with that of our previous studies, which indicated that the severity of PEA-induced hepatotoxicity was closely correlated with pro-inflammatory cytokines such as TNF- $\alpha[8,9]$.

A previous study has also shown that IL-2 stimulated the expression of pro-inflammatory cytokines/chemokines from liver nonparenchymal cells, such as T cells, Kupffer cells, and stellate cells, which contributes to liver inflammation [19]. The role of IL-2 was believed to be a pivotal mediator of liver injury in PEA-treated rats $[8,18,19]$. The present study showed that the PEA-induced serum 
levels of IL-2 directly correlated with the severity of hepatotoxicity, i.e., IL-2 values were the highest in BN/SsN, intermediate and variable in LE, WKY and LEW, and lowest in Wistar and F344 rats. This phenomenon showed that PEA treatment caused different levels of hepatotoxicity in different rat strains. In Con-A induced liver injury models, increased plasma IL-2 levels and T cell infiltration were observed [10,22]. Therefore, inhibition of the IL-2 signaling pathway might offer protection from this hepatotoxic effect [23]. A recent study has shown that inhibition of T cells producing IL-2 may prevent PEA-induced hepatotoxicity [19]. Therefore, we suggest that PEA-induced hepatotoxicity in different rat strains directly correlates with the capacity of IL-2 induction.

IL-6 is a cytokine involved not only in inflammation and infection responses, but also in the regulation of metabolic, regenerative, and neural processes [24]. In murine sepsis models, blockade of IL-6 trans-signaling was sufficient to rescue mice from death [25]. Blockade of IL-6 signaling aggravates liver injury and lethality in a D-galactosamine $\mathrm{N}(\mathrm{GalN})$ /LPS hepatitis model [26]. In our study, serum IL-6 levels were significantly higher in the $\mathrm{BN} / \mathrm{SsN}$ rats, but it was rarely detected, or detected at very low concentrations in Wistar and F344 strain rats. This phenomenon was consistent with our previous studies, indicating that the IL-6 dependent signal pathway may be involved in immune-mediated, PEA-induced hepatotoxicity.

IL-10 is a potent anti-inflammatory cytokine that inhibits the inflammatory reaction, initiation, and progress [27]. In this work, the serum levels of IL-10 increased significantly in the BN/SsN rats compared with other rat strains. This is presumably a consequence of the severe liver injury induced by PEA in BN/SsN rats. In contrast, the serum levels for IL-10 were low to moderate in other rat strains. Therefore, these results directly correlate with the degree of liver tissue injuries observed between the different rat strains.

Previous studies indicated the absence or reduce of LRP in different cell line, including $\mathrm{LRP}^{-/-}$embryonic fibroblasts, hepatoma and macrophage, were sufficient to protect cells from PEA intoxication [4-7]. These findings suggested that PEA might use LRP to gain entry into toxin-sensitive cells. In the present study, we compared the LRP expression in liver tissue from different rat strains. However, the results showed no significantly difference in LRP expression levels in each group. Based on these results, we suspect that the PEA-mediated differences in severity of liver injury we detected in different rat strains may not correlated with LRP expression.

In conclusion, we have demonstrated the differences in the genetic susceptibility to PEA-induced liver injury among four inbred strains (F344, WKY, BN/SsN and LEW) and two stocks (LE and Wistar) of rats, and found $\mathrm{BN} / \mathrm{SsN}$ rats to be the most sensitive. Our findings suggest that the genetic background plays an important role in the risk for hepatotoxicity in immune-mediated liver injury. In addition, strain differences in the susceptibility of PEA-induced liver injury may be influenced by the response to pro-inflammatory cytokines (TNF- $\alpha$, IL-2 and IL-6) that are induced as part of the inflammation process, which is in turn determined by the genetic background. Due to this, PEA-based immunotoxins for clinical use are being developed by many research teams [28,29]. Our data support the notion that variations in the immune response may be the most critical factor in PEA-induced hepatotoxicity. Hence, these results support the concept that variations in the genetic background of the host will manifest as different responses to diverse xenobiotic or biohazard intoxication.

\section{Materials and Methods}

Chemical and Animals. PEA was purchased from Calbiochem Chemical Company (La Jolla, CA, USA). A total of 72 specific-pathogen-free male LE, Wistar, F344, WKY, BN/SsN and LEW rats were purchased from BioLASCO Technology (Taipei, Taiwan) and National Laboratory Animal Center (Taipei, Taiwan), and used at 8-9 weeks of age. Animals were maintained at room temperature $\left(21 \pm 2{ }^{\circ} \mathrm{C}\right)$, with $55-65 \%$ relative humidity, and a $12 \mathrm{~h}$ light-dark cycle. Rats were fed a standard laboratory rat diet and provided with water ad libitum. All procedures were performed in an animal facility accredited by the Association for Assessment and Accreditation of Laboratory Animal Care 
(AAALAC) International, with the approval of the Institutional Animal Care and Use Committee (IACUC)(Ethical approval code: NLAC-94-R-001, Date of approval: 28 December 2005).

Experimental procedures. Different rat strains (eight per strain) were assigned to six groups. Rats were injected intravenously via the tail vein with $20 \mu \mathrm{g} / \mathrm{kg}$ PEA diluted in Dulbecco's phosphate-buffered saline (DPBS) (Gibco, Grand Island, NY, USA). For the purpose of minimizing animal use, the DPBS control groups contained four rats per strain for each rat strain. After $48 \mathrm{~h}$, the animals were euthanized by $\mathrm{CO} 2$ asphyxiation followed by exsanguination. Blood was withdrawn by cardiac puncture for analysis of clinical chemistry, complete blood count (CBC), and quantification of cytokines. Livers were excised and divided into several parts for various assays.

Clinical chemistry in blood and CBC. Serum samples were centrifuged at $2700 \mathrm{~g}$ for $10 \mathrm{~min}$. Liver function tests, including serum aspartate aminotransferase (AST), alanine aminotransferase (ALT), and icterus index were done using an automatic analyzer (HITACHI 717, Hitachi, Tokyo, Japan). Blood used for CBC was treated with EDTA. Total blood cells, differential leukocytes, and erythrocytes were measured using the Bayer Hematology System (ADVIA 2010, Bayer, NY, USA).

Histological examination and TUNEL staining. Liver fixation, processing, and embedding were performed as previously described [9]. Sagittal sections of the left lobe were obtained and stained with hematoxylin and eosin (H\&E) for histological examination. The scores, grading, and definition of hepatic lesions were: $0=$ no lesions, no necrosis; 1 = mild, single-cell necrosis; 2 = moderate, hepatocyte necrosis mostly around periportal areas; and $3=$ severe, extensive to massive necrosis [21]. The scoring and grading for hemorrhage was: $0=$ no hemorrhage; $1=$ mild hemorrhage; and $2=$ severe hemorrhage. The results of score was combined hepatic lesions and hemorrhage grading.

For the detection of hepatocyte apoptosis, $4 \mathrm{~mm}$ paraffin-embedded sections were deparaffinized, rehydrated, and stained with an apoptosis-specific staining kit (In Situ Cell Death/AP Detection Kit; Roche Diagnostics, Mannheim, Germany) according to the manufacturer's instructions. Slides were randomized, coded, and evaluated by a veterinary pathologist using light microscopy. The scores for the level of apoptosis were dependent on the number of TUNEL-positive cells per $100 \times$ magnification field: $0=$ no TUNEL-positive cells; $1=2-5$ TUNEL-positive cells; $2=6-10$ TUNEL-positive cells; and $3=$ more than 11 TUNEL-positive cells. This calculation method has been described previously.

Serum cytokine levels assay. Quantitative determinations of serum levels for TNF- $\alpha$, IL-2, IL-6 and IL-10 were performed using a specific ELISA kits (Invitrogen, Carlsbad, CA, USA). The ELISA assays were performed as detailed by the manufacturer.

Measurement of hepatic LRP expression levels. The liver tissue was homogenized immediately in extraction buffer using a handheld homogenizer (T 10 basic, IKA, Germany). The extracts were centrifuged at 11,000 rpm and $4 \mathrm{C}$ in a Sigma Centrifuge 4k15 (Ostrode am Harz, Germany) for $30 \mathrm{~min}$ to remove insoluble material, and the supernatants of these tissues were used for protein quantification, using the Bradford method. Aliquots of $0.2 \mathrm{mg}$ of protein extracts, obtained from each tissue, were separated by SDS-PAGE, transferred to nitrocellulose membranes and blotted with anti-LRP and anti- $\beta$-actin antibodies, from Santa Cruz Biotechnology (Santa Cruz, CA, USA). The original membrane was stripped and reblotted with $\beta$-actin loading protein. Chemiluminescent detection was performed with horseradish peroxidase-conjugate secondary antibodies. Visualization of protein bands was performed by exposure of membranes to RX films. Band intensities were quantified by optical densitometry (Scion Image software, version 4.0.3, ScionCorp, Frederick, MD, USA) of the developed autoradiographs.

Statistical analysis. Data including clinical chemistry and serum cytokines were presented as mean \pm standard deviation (mean $\pm \mathrm{SD}$ ). Significant differences between each treated group were determined by one-way analysis of variance (ANOVA) and post hoc Fisher's least significant differences (LSD) test by SPSS 18.0 software. Differences between groups were considered to be statistically significant $\left({ }^{*}\right)$ when $p<0.05$. 
Supplementary Materials: The following are available online at www.mdpi.com/2072-6651/9/7/224/s1, Figure S1. The expression fold change of the (A) ALT and (B) AST in the PEA-treated rat compared to DPBS-treated rat, Table S1. Clinical chemistry, complete blood count, cytokines of serum and score grading in the DPBS-treated rats.

Acknowledgments: This study was supported by a Grant from Ministry of Science and Technology (NSC 95-2313-B-492-001) of Taiwan.

Author Contributions: C.-C.C., Y.-C.W., Y.-C.C. conceived and designed the experiments; W.-C.H., Y.-H.C., S.-W.H. performed the experiments; Y.-T.H. and H.-L.C. analyzed the data; C.-C.C. and Y.-C.C. wrote the paper.

Conflicts of Interest: The authors declare no conflict of interest.

\section{References}

1. Williams, H.B.; Breidenbach, W.C.; Callaghan, W.B.; Richards, G.K.; Prentis, J.J. Are burn wound biopsies obsolete? A comparative study of bacterial quantitation in burn patients using the absorbent disc and biopsy techniques. Ann. Plast. Surg. 1984, 13, 388-395. [CrossRef] [PubMed]

2. Burns, J.L.; Emerson, J.; Stapp, J.R.; Yim, D.L.; Krzewinski, J.; Louden, L.; Ramsey, B.W.; Clausen, C.R. Microbiology of sputum from patients at cystic fibrosis centers in the United States. Clin. Infect. Dis. 1998, 27, 158-163. [CrossRef] [PubMed]

3. Tang, H.B.; DiMango, E.; Bryan, R.; Gambello, M.; Iglewski, B.H.; Goldberg, J.B.; Prince, A. Contribution of specific Pseudomonas aeruginosa virulence factors to pathogenesis of pneumonia in a neonatal mouse model of infection. Infect. Immun. 1996, 64, 37-43. [PubMed]

4. Kounnas, M.Z.; Morris, R.E.; Thompson, M.R.; FitzGerald, D.J.; Strickland, D.K.; Saelinger, C.B. The alpha 2-macroglobulin receptor/low density lipoprotein receptor-related protein binds and internalizes Pseudomonas exotoxin A. J. Biol. Chem. 1992, 267, 12420-12423. [PubMed]

5. Grimsley, P.G.; Quinn, K.A.; Chesterman, C.N.; Owensby, D.A. Low density lipoprotein receptor-related protein (LRP) expression varies among Hep G2 cell lines. Thromb. Res. 1997, 88, 485-498. [CrossRef]

6. Laithwaite, J.E.; Benn, S.J.; Yamate, J.; FitzGerald, D.J.; LaMarre, J. Enhanced macrophage resistance to Pseudomonas exotoxin A is correlated with decreased expression of the low-density lipoprotein receptor-related protein. Infect. Immun. 1999, 67, 5827-5833. [PubMed]

7. Laithwaite, J.E.; Benn, S.J.; Marshall, W.S.; FitzGerald, D.J.; LaMarre, J. Divergent Pseudomonas exotoxin A sensitivity in normal and transformed liver cells is correlated with low-density lipoprotein receptor-related protein expression. Toxicon 2001, 39, 1283-1290. [CrossRef]

8. Chiu, C.C.; Chen, H.H.; Chuang, H.L.; Chung, T.C.; Chen, S.D.; Huang, Y.T. Pseudomonas aeruginosa exotoxin A-induced hepatotoxicity: An animal model in rats. J. Vet. Med. Sci. 2009, 71, 1-8. [CrossRef] [PubMed]

9. Chuang, H.L.; Huang, Y.T.; Chiu, C.C.; Chen, H.H.; Chu, Y.Y.; Chen, T.H. Influence of age on susceptibility to Pseudomonas aeruginosa exotoxin A-induced hepatotoxicity in Long-Evans rats. J. Vet. Med. Sci. 2009, 71, 163-169. [CrossRef] [PubMed]

10. Mizuhara, H.; Kuno, M.; Seki, N.; Yu, W.G.; Yamaoka, M.; Yamashita, M.; Ogawa, T.; Kaneda, K.; Fujii, T.; Senoh, H.; et al. Strain difference in the induction of T-cell activation-associated, interferon gamma-dependent hepatic injury in mice. Hepatology 1998, 27, 513-519. [CrossRef] [PubMed]

11. Harrill, A.H.; Watkins, P.B.; Su, S.; Ross, P.K.; Harbourt, D.E.; Stylianou, I.M.; Boorman, G.A.; Russo, M.W.; Sackler, R.S.; Harris, S.C.; et al. Mouse population-guided resequencing reveals that variants in CD44 contribute to acetaminophen-induced liver injury in humans. Genome Res. 2009, 19, 1507-1515. [CrossRef] [PubMed]

12. Gonzalez, F.J. Role of cytochromes P450 in chemical toxicity and oxidative stress: Studies with CYP2E1. Mutat. Res. 2005, 569, 101-110. [CrossRef] [PubMed]

13. Fengler, V.H.; Macheiner, T.; Kessler, S.M.; Czepukojc, B.; Gemperlein, K.; Müller, R.; Kiemer, A.K.; Magnes, C.; Haybaeck, J.; Lackner, C.; et al. Susceptibility of Different Mouse Wild Type Strains to Develop Diet-Induced NAFLD/AFLD-Associated Liver Disease. PLoS ONE 2016, 11, e0155163. [CrossRef] [PubMed]

14. Mikus, L.D.; Rosenthal, L.A.; Sorkness, R.L.; Lemanske, R.F., Jr. Reduced interferon-gamma secretion by natural killer cells from rats susceptible to postviral chronic airway dysfunction. Am. J. Respir. Cell Mol. Biol. 2001, 24, 74-82. [CrossRef] [PubMed] 
15. Hines, E.A.; Szakaly, R.J.; Leng, N.; Webster, A.T.; Verheyden, J.M.; Lashua, A.J.; Kendziorski, C.; Rosenthal, L.A.; Gern, J.E.; Sorkness, R.L.; et al. Comparison of temporal transcriptomic profiles from immature lungs of two rat strains reveals a viral response signature associated with chronic lung dysfunction. PLoS ONE 2014, 9, e112997. [CrossRef] [PubMed]

16. Liu, J.; Sendelbach, L.E.; Parkinson, A.; Klaassen, C.D. Endotoxin pretreatment protects against the hepatotoxicity of acetaminophen and carbon tetrachloride: Role of cytochrome P450 suppression. Toxicology 2000, 147, 167-176. [CrossRef]

17. Chiu, C.C.; Huang, Y.T.; Wang, Y.C.; Chang, Y.C.; Ching, Y.H.; Chen, H.H.; Chuang, H.L. Pretreatment with lipopolysaccharide ameliorates Pseudomonas exotoxin A-induced hepatotoxicity in rats. Immunopharmacol. Immunotoxicol. 2013, 35, 296-303. [CrossRef] [PubMed]

18. Faggioni, R.; Jones-Carson, J.; Reed, D.A.; Dinarello, C.A.; Feingold, K.R.; Grunfeld, C.; Fantuzzi, G. Leptin-deficient $(\mathrm{ob} / \mathrm{ob})$ mice are protected from $\mathrm{T}$ cell-mediated hepatotoxicity: Role of tumor necrosis factor alpha and IL-18. Proc. Natl. Acad. Sci. USA 2000, 97, 2367-2372. [CrossRef] [PubMed]

19. Mühlen, K.A.; Schümann, J.; Wittke, F.; Stenger, S.; Van Rooijen, N.; Van Kaer, L.; Tiegs, G. NK cells, but not NKT cells, are involved in Pseudomonas aeruginosa exotoxin A-induced hepatotoxicity in mice. J. Immunol. 2004, 172, 3034-3041. [CrossRef] [PubMed]

20. Schümann, J.; Mühlen, K.; Kiemer, A.K.; Vollmar, A.M.; Tiegs, G. Parenchymal, but not leukocyte, TNF receptor 2 mediates $\mathrm{T}$ cell-dependent hepatitis in mice. J. Immunol. 2003, 170, 2129-2137. [CrossRef] [PubMed]

21. Chiu, C.C.; Huang, Y.T.; Chuang, H.L.; Chen, H.H.; Chung, T.C. Co-exposure of lipopolysaccharide and Pseudomonas aeruginosa exotoxin A-induced multiple organ injury in rats. Immunopharmacol. Immunotoxicol. 2009, 31, 75-82. [CrossRef] [PubMed]

22. Nicoletti, F.; Beltrami, B.; Raschi, E.; Di Marco, R.; Magro, G.; Grasso, S.; Bendtzen, K.; Fiorelli, G.; Meroni, P.L. Protection from concanavalin A (Con A)-induced T cell-dependent hepatic lesions and modulation of cytokine release in mice by sodium fusidate. Clin. Exp. Immunol. 1997, 110, 479-484. [CrossRef] [PubMed]

23. Gantner, F.; Leist, M.; Lohse, A.W.; Germann, P.G.; Tiegs, G. Concanavalin A-induced T-cell-mediated hepatic injury in mice: The role of tumor necrosis factor. Hepatology 1995, 21, 190-198. [PubMed]

24. Scheller, J.; Chalaris, A.; Schmidt-Arras, D.; Rose-John, S. The pro- and anti-inflammatory properties of the cytokine interleukin-6. Biochim. Biophys. Acta. 2011, 1813, 878-888. [CrossRef] [PubMed]

25. Barkhausen, T.; Tschernig, T.; Rosenstiel, P.; van Griensven, M.; Vonberg, R.P.; Dorsch, M.; Mueller-Heine, A.; Chalaris, A.; Scheller, J.; Rose-John, S.; et al. Selective blockade of interleukin-6 trans-signaling improves survival in a murine polymicrobial sepsis model. Crit. Care Med. 2011, 39, 1407-1413. [CrossRef] [PubMed]

26. Barton, B.E.; Jackson, J.V. Protective role of interleukin 6 in the lipopolysaccharide-galactosamine septic shock model. Infect. Immun. 1993, 61, 1496-1499. [PubMed]

27. Thompson, K.; Maltby, J.; Fallowfield, J.; McAulay, M.; Millward-Sadler, H.; Sheron, N. Interleukin-10 expression and function in experimental murine liver inflammation and fibrosis. Hepatology 1998, 28, 1597-1606. [CrossRef] [PubMed]

28. Wang, L.F.; Zhou, Y.; Xu, Y.M.; Qiu, X.C.; Zhou, B.G.; Wang, F.; Long, H.; Chen, X.; Yang, T.T.; Ma, B.A.; et al. A caspase- 6 and anti-HER2 antibody chimeric tumor-targeted proapoptotic molecule decreased metastasis of human osteosarcoma. Cancer Investig. 2009, 27, 774-780. [CrossRef] [PubMed]

29. Borowiec, M.; Gorzkiewicz, M.; Grzesik, J.; Walczak-Drzewiecka, A.; Salkowska, A.; Rodakowska, E.; Steczkiewicz, K.; Rychlewski, L.; Dastych, J.; Ginalski, K. Towards Engineering Novel PE-Based Immunotoxins by Targeting Them to the Nucleus. Toxins (Basel) 2016, 8. [CrossRef] [PubMed]

(C) 2017 by the authors. Licensee MDPI, Basel, Switzerland. This article is an open access article distributed under the terms and conditions of the Creative Commons Attribution (CC BY) license (http://creativecommons.org/licenses/by/4.0/). 